\title{
Assessing the Influence of Parental Anxiety on Child Anxiety During the COVID-19 Pandemic in the United Arab Emirates
}

\author{
Basema Saddik \\ University of Sharjah College of Medicine https://orcid.org/0000-0002-4682-5927 \\ Amal Hussein \\ University of Sharjah College of Medicine \\ Ammar Albanna \\ Al Jalila Children's Specialty Hospital \\ Iffat Elbarazi \\ United Arab Emirates University College of Medicine and Health Sciences \\ Arwa Al-Shujairi \\ University of Sharjah \\ Fatemeh S. Sharif-Askari \\ University of Sharjah College of Medicine \\ Mohamad Hani Temsah \\ King Saud University College of Medicine \\ Emmanuel Stip \\ United Arab Emirates University College of Medicine and Health Sciences \\ Qutayba Hamid \\ University of Sharjah College of Medicine \\ Rabih Halwani ( $\square$ rhalwani@sharjah.ac.ae) \\ University of Sharjah, Sharjah
}

Research article

Keywords: COVID-19, anxiety, children, United Arab Emirates

Posted Date: October 5th, 2020

DOI: https://doi.org/10.21203/rs.3.rs-66841/v1

License: (c) (1) This work is licensed under a Creative Commons Attribution 4.0 International License. Read Full License 


\section{Abstract}

Background: Studies have investigated the psychosocial impact of infectious disease outbreaks in adults; however, there is limited information on the mental health impact of the COVID-19 pandemic on children and the general UAE community. The aim of this study was to explore anxiety levels among parents, teachers and the general community amid the COVID-19 pandemic in the UAE, as well as to identify emotional and anxiety disorders in children.

Methods: Using a web-based cross-sectional survey we collected data from 2,200 self-selected, assessed volunteers. Demographic information, knowledge and beliefs about COVID-19, generalized anxiety disorder (GAD) using the (GAD-7) scale, emotional problems in children using the strengths and difficulties questionnaire (SDQ), worry and fear about COVID-19, coping mechanisms and general health information were collected.

Results: The overall prevalence of GAD in the general population was 71\% with younger people (59.8\%) and females (51.7\%) reporting highest levels of anxiety. Parents who were teachers reported the highest percentage of emotional problems in children (26.7\%). Multivariate logistic regression for GAD-7 scores showed that being female, reporting high levels of worry associated with COVID-19, intention to take the COVID-19 vaccine and smoking were associated with higher levels of anxiety. Multivariate logistic regression for SDQ showed that parents with severe anxiety were seven times more likely to report emotional problems in their children than less anxious parents.

Conclusions: This study is among the first to report the psychological impact of COVID-19 among adults and children in the UAE, and to highlight the significant association between parental and child anxiety. Findings suggest the urgency for policy makers to develop effective screening and coping strategies for parents and especially children.

\section{Background}

The coronavirus disease 2019 (COVID-19) emerged in Wuhan, China, December 2019, and was declared a public health emergency on January 30th [1] and a global pandemic by the WHO on March 11th [2]. By June 16th, more than 8 million confirmed cases and over 440,000 deaths were reported worldwide, with 42,982 confirmed cases and 293 deaths in the UAE [3]. In the absence of an effective treatment or vaccine, unprecedented public health interventions were implemented across the UAE to curb transmission of the disease. These included international border closures, travel bans, lockdowns, closures of schools and universities, strict social distancing and quarantines. These measures, along with fear of the pandemic and disruption in people's lives have significant mental health implications [4].

Research on past infectious disease outbreaks, such as severe acute respiratory syndrome (SARS), swine flu, and influenza revealed a wide range of psychosocial impacts at individual, community, and international levels. These included worries about infection and fear of dying [5], increase in anxiety, post-traumatic stress and depression [6], feelings of helplessness and panic and increased perception of risk [7-9]. Studies investigating the psychological impacts of COVID-19 in China reported moderate to severe stress, generalized anxiety, insomnia, and depression [10, 11].

Information about the mental health impact of COVID-19 on the general population, especially children, is limited, and there are no published reports on anxiety levels in the UAE. While severe COVID-19 in children is less frequent than in adults [12], the mental health of children may be disproportionately affected by changes in their routines, reduction in social contact and anxiety. With the recent evidence on how parental anxiety can cause anxiety disorders in children $[13,14]$ and reports on the psychological impact of COVID-19 on adults, the mental well-being of children should not be ignored. Parent and teacher observations are important in screening children for emotional disorders $[15,16]$. As observers, parents and teachers can positively influence children's well-being $[17,18]$.

Here we explored anxiety among parents, teachers and the general community amid the COVID-19 pandemic in the UAE, as well as anxiety disorders in children as reported by parents and teachers. We assessed parents' and teachers' knowledge, beliefs and risk perceptions related to COVID-19, health-protection and hygiene, worries and fears and coping mechanisms to reduce children's anxiety. This is the first study in the UAE to discuss this issue. 


\section{Methods}

\subsection{Participants}

Convenience sampling was used to recruit participants. We randomly selected and contacted 17 schools across the UAE to participate in the study. However, with closures and online learning, only four schools responded. Participants were invited to take an online survey using email announcements through participating schools and universities and posts on Facebook, Instagram and WhatsApp. Teachers, parents, and members of the public throughout the UAE, 18 years and older, participated and passed the survey link to friends. Data were collected from 24th March to 15th May, 2020. The survey was administered via the Survey Monkey platform [19], and each response came from a unique IP address to ensure unique entries. The first page explained the research objectives and assured confidentiality. The minimum sample size needed for this cross-sectional study was 385 , calculated for an expected prevalence of $50 \%$, margin of error of $5 \%$, and $95 \%$ confidence.

\subsection{Ethical approval and consent}

The study was approved by the University of Sharjah Ethics Committee (approval number REC-20-03-12-01) and the United Arab Emirates University research ethics review board (ERS_2020_6098) and all research was performed in accordance with regulations of these committees. Participants gave written consent to participate in the study before beginning the survey.

\subsection{Data collection}

A structured questionnaire comprising 32 items was used. Questions were divided into eight domains: demographics, knowledge, beliefs and perceived risk related to COVID-19, health-protective and hygienic behaviors, precautionary measures, worry and fear associated with COVID-19, general health, validated self-reported anxiety screening scales (adults and children) and coping mechanisms. The questionnaire (Appendix 1) was translated into Arabic by a certified translator, and back-translated to English to ensure accuracy. The final version was piloted among ten members of the general community to ensure clarity and consistency. The questionnaire was sent to a group of ten experts consisting of faculty, teachers, parents, and a mental health expert who reviewed the survey for accuracy, length, clarity and comprehensiveness. Modifications were made to questions and response items based on expert recommendations. The questionnaire took ten minutes to complete.

\section{Demographics}

Information was collected on participants' age, gender, educational level, emirate or country of residence, marital status, number of children, ages and level of schooling, employment status, monthly income and health insurance. Participants indicated if they were parents, a parent and teacher, teachers only or neither parents nor teachers.

Knowledge, beliefs and perceived risks related to COVID-19

Participants were asked to answer "true", "false", or "don't know" on statements related to COVID-19, such as "there is no specific treatment" and "I feel a sense of social responsibility by staying at home". Perceived risk from COVID-19 was assessed on a 4point Likert scale (very likely to not likely at all) where participants responded to the likelihood of contracting COVID-19, surviving COVID-19 or developing severe illness.

Health-protective practices and hygienic behaviors

Participants described how often they followed hygienic measures. Responses to seven questions (covering mouth when sneezing/coughing, using hand sanitizer, hand-washing, wearing facemasks, avoiding crowded areas, public transport and handshakes) were measured on a 4-point Likert scale (always to never). These questions were modified from versions used in studies during MERS-CoV, swine flu and SARS $[7,9,20,21]$. to categorize hygienic behavior into dichotomous types a standard median split was performed [22] with a median cut-off of 25. A value of $\geq 25$ indicated high exhibiting behaviors.

Worry and fear associated with COVID-19

Page $3 / 24$ 
To assess worry and fear of COVID-19, participants were asked to rate how worried they were on seven questions: Worried about catching COVID-19 myself; Worried about parents catching COVID-19; Worried about child catching COVID-19; Worried about what COVID-19 can do to me health-wise;Worried about social isolation/quarantine; Worried about loss of income; and Worried about transmitting the virus to family and friends. Participants responded on a 5-point Likert scale from extremely worried to not worried at all. Participants were also asked their opinion on public fear associated with COVID-19 on a 5-point Likert scale (strongly agree to strongly disagree) [23]. To categorize worry into dichotomous categories, a standard median split was carried out [22] with a median cutoff of 22 . A value of $\geq 22$ was signified very worried.

\section{Anxiety}

Anxiety among adults was measured using the generalized anxiety disorder scale (GAD-7) [24] which is a self-reported 7-item validated scale. Participants indicated how often they were bothered during the previous two weeks by symptoms of feeling nervous, not being able to stop worrying, worrying about different things, trouble relaxing, restless, irritable and afraid that something awful might happen. Response options were "not at all," "several days," "more than half the days," and "nearly every day," scored as $0,1,2$, and 3 . A score of $\geq 10$ identified cases of anxiety with $89 \%$ sensitivity and $82 \%$ specificity, good internal consistency (Cronbach $a=.92$ ) and test-retest reliability (intraclass correlation $=0.83$ ) [24]. Other research established a cutoff of 8 , (sensitivity $77 \%$, specificity $82 \%$ ) as a screener for panic disorder, social anxiety phobia and PTSD [25]. GAD-7 scores were totaled and classified as minimal (0-4), mild (5-9), moderate (10-14) and severe (15-21) [24], and stratified into two groups (< 8 or $>8)$ as a cut-off for panic disorder and social anxiety phobia [25].

Children's anxiety levels were measured using the emotional symptoms sub-scale from the strengths and difficulties questionnaire (SDQ) [26], which covers emotional symptoms, conduct problems, hyperactivity-inattention, peer relationship problems, and prosocial behaviors. It was designed to screen for psychological disorders in children aged 3 to 16 years [16]. The emotional symptoms sub-scale [27] asks parents and teachers questions about symptoms they have witnessed in children: Often complains of headaches, stomach-ache or sickness; Many worries, often seems worried; Often unhappy, down-hearted or tearful; Nervous or clingy in new situations, easily loses confidence; and, Many fears, easily scared. Each item can be marked "not true" (0), "somewhat true" (1) or "certainly true" (2) thereby generating a score of 0-10. A cutoff score of 7 indicates generalized anxiety disorder (sensitivity $75 \%$, specificity $80 \%$ ) and depressive and generalized anxiety disorders (sensitivity $67 \%$, specificity $81 \%$ ) [28]. According to scoring guidelines [29], an abnormal emotional problems score completed by both parents and teachers ranged from 5-10 and SDQ $\geq 5$ was used as an indicator of abnormal emotional problems. Validated Arabic translations of both the GAD-7 and SDQ were used for the Arabic translation of the questionnaire [30, 31].

To determine the impact of measures to reduce anxiety, participants were asked whether they felt less anxious with the introduction of online learning, airport screening, travel bans, availability of hand sanitizer in public places, cancellation of social events, temporary closure of public places and social isolation. Responses were recorded on a 5-point Likert scale (strongly agree to strongly disagree). To categorize precautionary measures into dichotomous categories, a standard median split was carried out [22] with a median cutoff of 34 . A score of $\geq 34$ indicated high agreement with precautionary measures.

\section{Coping mechanisms}

Participants were asked to indicate on a 4-point Likert scale (always to never) which coping mechanisms they used to reduce anxiety in their children and family. Questions included: openly discussing COVID-19 with children/family, educating children about proper hygiene, assuring children they are safe, limiting children's exposure to news coverage and social media, creating a schedule of learning and fun activities and maintaining regular routine. To categorize coping strategies into dichotomous categories a standard median split was carried out [22] with a median cutoff of 18 . A score of $\geq 18$ indicated high coping strategies.

\section{General health}

Participants were asked whether they suffered from chronic disease or had flu-like symptoms over the previous two weeks, the treatments for such symptoms, the likelihood of taking a COVID-19 vaccine, whether their children were vaccinated, whether they smoked and if their smoking habits had changed since the outbreak. 


\subsection{Statistical analysis}

Descriptive statistics, including means, medians, frequencies and percentages were used to summarize data and to illustrate participants' demographics and characteristics. The normal distribution of data was verified visually using histograms, boxplots, and quantile-quantile plots, and statistically using the Kolmogorov-Smirnov test. The equality of variances was checked using Levene's test. Bivariate analysis used Chi-squared $(\chi 2)$ tests to explore associations between participant demographics and anxiety levels. Statistically significant factors in the bivariate analysis were included in multivariate binary logistic regression models to determine predictors of anxiety levels (GAD-7 score $\geq 8$ ) and emotional problems in children (SDQ score $\geq 5$ ). The automatic selection of predictors in the model was performed by a stepwise backward method with an entry threshold of 0.05 and an exit threshold of 0.1 . The adequacy of the model was verified by the Hosmer and Lemeshow test and the specificity of the model by Link Test. The estimates of the strengths of associations were demonstrated by the odds ratio (OR) with a $95 \%$ confidence interval $(\mathrm{Cl})$. A two-tailed $p<0.05$ was considered statistically significant. Data were analyzed using statistical software SAS® 9.3 [32].

\section{Results}

In total, 2200 people completed the online participant sheet and consent form. Of these, 26 declined to participate and 381 completed only the demographic part of the questionnaire before discontinuing. Complete data were analyzed for 1469 participants (68\%). Table 1 summarizes the demographics. Participants were primarily female (82.8\%), 25 to 44 years of age $(61.7 \%)$ and resided in the UAE (72.8\%). Over half of our population held a bachelor's degree $(50.8 \%)$ and were employed (63.1\%). Seventy five percent of participants were married and had children (75.6\%), with the majority having 1-2 children (35.2\%). The most commonly reported medical conditions were high blood pressure (9.1\%) and asthma (8.6\%). Headaches (25.9\%) were the most commonly reported COVID-19 symptom and almost half of participants indicated they used vitamin C for their symptoms. Whilst most participants reported they did not smoke, $13.7 \%$ stated they had changed their smoking habits since the COVID-19 outbreak. Most indicated they would get vaccinated (71.5\%) and have their children vaccinated (59.4\%) against COVID-19. The majority indicated their children were current with vaccinations (85\%); however, we found a significant association between those who reported their children were not current with vaccinations $(53 \%)$ and their intention to not vaccinate their children against COVID-19. 
Demographic characteristics by anxiety score (GAD-7) and children emotional SDQ score $(n=1469)$

\begin{tabular}{|c|c|c|c|c|c|c|c|c|c|}
\hline \multicolumn{4}{|c|}{ Demographics } & \multicolumn{3}{|c|}{ Anxiety GAD-7 Score $(\geq 8)$} & \multicolumn{3}{|c|}{$\begin{array}{l}\text { Reported Children Emotional } \\
\text { SDQ Score (SDQ } \geq 5)\end{array}$} \\
\hline Variable & Category & $\begin{array}{l}\text { Frequency } \\
\text { (n) }\end{array}$ & $\%$ & $\%(n)$ & $\begin{array}{l}\text { Chi- } \\
\text { square }\end{array}$ & $\begin{array}{l}\mathrm{p}- \\
\text { value }\end{array}$ & $\%(n)$ & $\begin{array}{l}\text { Chi- } \\
\text { square }\end{array}$ & $\begin{array}{l}\mathrm{p}- \\
\text { value }\end{array}$ \\
\hline \multirow[t]{2}{*}{ Gender } & Female & 1216 & 82.8 & $\begin{array}{l}51.7 \\
(629)\end{array}$ & 10.16 & $0.001^{*}$ & $\begin{array}{l}16.2 \\
(154)\end{array}$ & 2.83 & 0.093 \\
\hline & Male & 253 & 17.2 & $\begin{array}{l}40.7 \\
(103)\end{array}$ & & & $11.5(24)$ & & \\
\hline \multirow[t]{4}{*}{$\begin{array}{l}\text { Relationship } \\
\text { to child/ren }\end{array}$} & Parent & 893 & 60.8 & $\begin{array}{l}48.2 \\
(430)\end{array}$ & 4.24 & 0.120 & $\begin{array}{l}14.6 \\
(130)\end{array}$ & 25.6 & $<0.001^{*}$ \\
\hline & Teacher only & 106 & 7.2 & $44.3(47)$ & & & $4.7(5)$ & & \\
\hline & Parent \& teacher & 161 & 11.0 & $55.9(90)$ & & & $26.7(43)$ & & \\
\hline & No children & 299 & 20.4 & $\begin{array}{l}54.2 \\
(162)\end{array}$ & & & 0 & & \\
\hline \multirow[t]{4}{*}{ Age } & $18-24$ & 169 & 11.5 & $\begin{array}{l}59.8 \\
(101)\end{array}$ & 22.27 & $\begin{array}{l}< \\
0.001^{*}\end{array}$ & $8.7(2)$ & 3.44 & 0.329 \\
\hline & $25-44$ & 907 & 61.7 & $\begin{array}{l}51.0 \\
(463)\end{array}$ & & & $\begin{array}{l}14.7 \\
(115)\end{array}$ & & \\
\hline & $45-64$ & 381 & 25.9 & $\begin{array}{l}43.6 \\
(166)\end{array}$ & & & $17.4(61)$ & & \\
\hline & $65+$ & 12 & 0.8 & $16.7(2)$ & & & 0 & & \\
\hline \multirow[t]{5}{*}{ Education } & Primary & 11 & 0.7 & $36.4(4)$ & 3.14 & 0.534 & $11.1(1)$ & 1.76 & 0.779 \\
\hline & Lower secondary & 10 & 0.7 & $30.0(3)$ & & & $25.0(2)$ & & \\
\hline & Higher secondary & 137 & 9.3 & $53.3(73)$ & & & $18.2(18)$ & & \\
\hline & Bachelor degree & 746 & 50.8 & $\begin{array}{l}50.1 \\
(374)\end{array}$ & & & $14.4(79)$ & & \\
\hline & Post-graduate & 565 & 38.5 & $\begin{array}{l}49.2 \\
(278)\end{array}$ & & & $15.8(78)$ & & \\
\hline \multirow[t]{2}{*}{$\begin{array}{l}\text { Country of } \\
\text { Residence }\end{array}$} & Outside the UAE & 400 & 27.2 & $\begin{array}{l}49.0 \\
(196)\end{array}$ & 0.15 & 0.697 & $15.6(51)$ & 0.02 & 0.882 \\
\hline & Inside the UAE & 1069 & 72.8 & $\begin{array}{l}50.1 \\
(536)\end{array}$ & & & $\begin{array}{l}15.2 \\
(127)\end{array}$ & & \\
\hline \multirow[t]{3}{*}{ Employment } & Employed & 927 & 63.1 & $\begin{array}{l}48.8 \\
(452)\end{array}$ & 2.00 & 0.372 & $\begin{array}{l}15.6 \\
(122)\end{array}$ & 1.08 & 0.583 \\
\hline & Not employed & 319 & 21.7 & $\begin{array}{l}53.3 \\
(170)\end{array}$ & & & $12.9(22)$ & & \\
\hline & Home duties & 223 & 15.2 & $\begin{array}{l}49.3 \\
(110)\end{array}$ & & & $16.6(34)$ & & \\
\hline \multirow{3}{*}{$\begin{array}{l}\text { Monthly } \\
\text { Salary }\end{array}$} & Other & 192 & 13.1 & $46.4(89)$ & 5.19 & 0.393 & $15.2(25)$ & 9.90 & 0.079 \\
\hline & less than 5,000 & 161 & 11.0 & $55.9(90)$ & & & $12.4(13)$ & & \\
\hline & $5,000-9,999$ & 163 & 11.1 & $54.0(88)$ & & & $20.2(23)$ & & \\
\hline
\end{tabular}




\begin{tabular}{|c|c|c|c|c|c|c|c|c|c|}
\hline & \multicolumn{3}{|l|}{ Demographics } & \multicolumn{3}{|c|}{ Anxiety GAD-7 Score $(\geq 8)$} & \multicolumn{3}{|c|}{$\begin{array}{l}\text { Reported Children Emotional } \\
\text { SDQ Score (SDQ } \geq 5)\end{array}$} \\
\hline & $10,000-19,999$ & 282 & 19.2 & $\begin{array}{l}48.9 \\
(138)\end{array}$ & & & $15.8(35)$ & & \\
\hline & $20,000-39,000$ & 413 & 28.1 & $\begin{array}{l}49.6 \\
(205)\end{array}$ & & & $18.1(60)$ & & \\
\hline & $40,000+$ & 258 & 17.6 & $\begin{array}{l}47.3 \\
(122)\end{array}$ & & & $9.9(22)$ & & \\
\hline \multirow[t]{3}{*}{ Insurance } & Other & 21 & 1.4 & $47.6(10)$ & 0.634 & 0.729 & $15.4(2)$ & 4.13 & 0.126 \\
\hline & No & 226 & 15.4 & $\begin{array}{l}52.2 \\
(118)\end{array}$ & & & $10.1(17)$ & & \\
\hline & Yes & 1222 & 83.2 & $\begin{array}{l}49.4 \\
(604)\end{array}$ & & & $\begin{array}{l}16.2 \\
(159)\end{array}$ & & \\
\hline \multirow[t]{4}{*}{$\begin{array}{l}\text { Marital } \\
\text { Status }\end{array}$} & Single & 287 & 19.5 & $\begin{array}{l}55.7 \\
(160)\end{array}$ & 7.27 & 0.064 & $4.2(3)$ & 11.9 & $0.008^{*}$ \\
\hline & Married & 1094 & 74.5 & $\begin{array}{l}47.8 \\
(523)\end{array}$ & & & $\begin{array}{l}15.5 \\
(157)\end{array}$ & & \\
\hline & $\begin{array}{l}\text { Divorced/ } \\
\text { Separated }\end{array}$ & 72 & 4.9 & $56.9(41)$ & & & $25.4(16)$ & & \\
\hline & Widowed & 16 & 1.1 & $50.0(8)$ & & & $14.3(2)$ & & \\
\hline \multirow[t]{2}{*}{ Children } & Yes & 1111 & 75.6 & $\begin{array}{l}49.1 \\
(545)\end{array}$ & 1.10 & 0.296 & & & \\
\hline & No & 358 & 24.4 & $\begin{array}{l}52.2 \\
(187)\end{array}$ & & & & & \\
\hline \multirow[t]{3}{*}{$\begin{array}{l}\text { Children } \\
\text { Number }\end{array}$} & 1-2 children & 517 & 35.2 & $\begin{array}{l}49.7 \\
(257)\end{array}$ & 4.85 & 0.089 & $16.6(80)$ & 0.070 & 0.966 \\
\hline & 3-4 children & 473 & 32.2 & $\begin{array}{l}50.5 \\
(239)\end{array}$ & & & $16.0(73)$ & & \\
\hline & $4+$ children & 119 & 8.1 & $39.5(47)$ & & & $16.5(19)$ & & \\
\hline \multirow[t]{5}{*}{$\begin{array}{l}\text { Age category } \\
\text { of children }\end{array}$} & $\begin{array}{l}\text { Infants and } \\
\text { Toddlers }\end{array}$ & 269 & 18.3 & $\begin{array}{l}52.4 \\
(141)\end{array}$ & 0.88 & 0.348 & $\begin{array}{l}16.2 \\
(154)\end{array}$ & 0.167 & 0.683 \\
\hline & Preschoolers & 325 & 22.1 & $\begin{array}{l}53.8 \\
(175)\end{array}$ & 2.69 & 0.101 & $11.5(24)$ & 0.085 & 0.770 \\
\hline & School Age & 713 & 48.5 & $\begin{array}{l}50.5 \\
(360)\end{array}$ & 0.24 & 0.623 & $\begin{array}{l}14.6 \\
(130)\end{array}$ & 5.93 & $0.015^{\star}$ \\
\hline & Adolescents & 432 & 29.4 & $\begin{array}{l}48.6 \\
(210)\end{array}$ & 0.36 & 0.547 & $\begin{array}{l}17.5 \\
(121)\end{array}$ & 8.83 & $0.003^{*}$ \\
\hline & Young Adults & 254 & 17.3 & $\begin{array}{l}42.5 \\
(108)\end{array}$ & 6.56 & $0.010^{*}$ & $19.6(81)$ & 1.53 & 0.216 \\
\hline \multirow{3}{*}{$\begin{array}{l}\text { Children } \\
\text { attending } \\
\text { school }\end{array}$} & $\begin{array}{l}\text { Children don't go to } \\
\text { school }\end{array}$ & 183 & 12.5 & $\begin{array}{l}55.7 \\
(102)\end{array}$ & 2.92 & 0.088 & $13.6(22)$ & 0.45 & 0.502 \\
\hline & Childcare & 205 & 14.0 & $\begin{array}{l}50.2 \\
(103)\end{array}$ & 0.01 & 0.898 & $16.4(32)$ & 0.20 & 0.651 \\
\hline & Primary & 668 & 45.5 & $\begin{array}{l}52.4 \\
(350)\end{array}$ & 3.23 & 0.073 & $\begin{array}{l}16.8 \\
(109)\end{array}$ & 2.39 & 0.123 \\
\hline
\end{tabular}




\begin{tabular}{|c|c|c|c|c|c|c|c|c|c|}
\hline & \multicolumn{3}{|l|}{ Demographics } & \multicolumn{3}{|c|}{ Anxiety GAD-7 Score $(\geq 8)$} & \multicolumn{3}{|c|}{$\begin{array}{l}\text { Reported Children Emotional } \\
\text { SDQ Score }(S D Q \geq 5)\end{array}$} \\
\hline & Lower secondary & 400 & 27.2 & $49(196)$ & 0.15 & 0.697 & $21.4(82)$ & 16.2 & $\begin{array}{l}< \\
0.001^{*}\end{array}$ \\
\hline & Higher Secondary & 320 & 21.8 & $\begin{array}{l}45.6 \\
(146)\end{array}$ & 2.89 & 0.089 & $20.5(63)$ & 8.61 & $0.003^{*}$ \\
\hline & University & 258 & 17.6 & $\begin{array}{l}42.6 \\
(110)\end{array}$ & 6.48 & $0.011^{*}$ & $17.4(40)$ & 0.92 & 0.336 \\
\hline \multirow{2}{*}{$\begin{array}{l}\text { Likely to } \\
\text { vaccinate } \\
\text { self }\end{array}$} & No & 383 & 26.1 & $\begin{array}{l}41.3 \\
(158)\end{array}$ & 20.05 & $\begin{array}{l}< \\
0.001^{*}\end{array}$ & & & \\
\hline & Yes & 1050 & 71.5 & $\begin{array}{l}53.2 \\
(559)\end{array}$ & & & & & \\
\hline \multirow{2}{*}{$\begin{array}{l}\text { Likely to } \\
\text { vaccinate } \\
\text { children }\end{array}$} & No & 314 & 21.4 & $\begin{array}{l}41.7 \\
(131)\end{array}$ & 20.71 & $0.001^{*}$ & & & \\
\hline & Yes & 872 & 59.4 & $\begin{array}{l}52.4 \\
(457)\end{array}$ & & & & & \\
\hline \multirow{8}{*}{$\begin{array}{l}\text { Medical } \\
\text { Conditions }\end{array}$} & Diabetes & 75 & 5.1 & $53.3(40)$ & 0.39 & 0.533 & & & \\
\hline & Heart Problems & 36 & 2.5 & $58.3(21)$ & 1.07 & 0.301 & & & \\
\hline & $\begin{array}{l}\text { High Blood } \\
\text { Pressure }\end{array}$ & 133 & 9.1 & $51.9(69)$ & 0.25 & 0.620 & & & \\
\hline & Dyslipidemia & 45 & 3.1 & $48.9(22)$ & 0.89 & 0.898 & & & \\
\hline & Asthma & 127 & 8.6 & $62.2(79)$ & 8.52 & $0.004^{*}$ & & & \\
\hline & $\begin{array}{l}\text { Respiratory } \\
\text { problems }\end{array}$ & 47 & 3.2 & $53.2(25)$ & 0.64 & 0.639 & & & \\
\hline & Cancer & 9 & 0.6 & $44.4(4)$ & 0.75 & 0.746 & & & \\
\hline & $\begin{array}{l}\text { Other medical } \\
\text { conditions }\end{array}$ & 128 & 8.7 & $50.8(65)$ & 0.82 & 0.822 & & & \\
\hline \multirow[t]{10}{*}{ Symptoms } & Headaches & 381 & 25.9 & $\begin{array}{l}56.7 \\
(216)\end{array}$ & 9.69 & $0.002^{*}$ & & & \\
\hline & Fever & 105 & 7.1 & $56.2(59)$ & 1.83 & 0.176 & & & \\
\hline & Cough & 235 & 16.0 & $\begin{array}{l}51.1 \\
(120)\end{array}$ & 0.17 & 0.680 & & & \\
\hline & Difficulty breathing & 37 & 2.5 & $51.4(19)$ & 0.03 & 0.851 & & & \\
\hline & Sore throat & 256 & 17.4 & $\begin{array}{l}59.8 \\
(153)\end{array}$ & 12.2 & $\begin{array}{l}< \\
0.001^{*}\end{array}$ & & & \\
\hline & Myalgia & 49 & 3.3 & $59.2(29)$ & 1.77 & 0.183 & & & \\
\hline & Dizziness & 73 & 5.0 & $63(46)$ & 5.34 & $0.021^{*}$ & & & \\
\hline & Runny nose & 225 & 15.3 & $\begin{array}{l}53.8 \\
(121)\end{array}$ & 1.66 & 0.198 & & & \\
\hline & Diarrhea & 100 & 6.8 & $50(50)$ & 0.001 & 0.972 & & & \\
\hline & Other Symptoms & 18 & 1.2 & $50(9)$ & $<0.001$ & 0.988 & & & \\
\hline
\end{tabular}




\begin{tabular}{|c|c|c|c|c|c|c|c|c|c|}
\hline \multicolumn{3}{|c|}{ Demographics } & \multicolumn{4}{|c|}{ Anxiety GAD-7 Score $(\geq 8)$} & \multicolumn{3}{|c|}{$\begin{array}{l}\text { Reported Children Emotional } \\
\text { SDQ Score (SDQ } \geq 5)\end{array}$} \\
\hline \multirow[t]{6}{*}{$\begin{array}{l}\text { Measures } \\
\text { taken }\end{array}$} & Vitamin C & 720 & 49.0 & $\begin{array}{l}53.5 \\
(385)\end{array}$ & 7.49 & $0.006^{*}$ & & & \\
\hline & Flu medications & 92 & 6.3 & $53.3(49)$ & 0.46 & 0.497 & & & \\
\hline & $\begin{array}{l}\text { Anti-inflammatory } \\
\text { drugs }\end{array}$ & 139 & 9.5 & $62.6(87)$ & 1.00 & $0.002^{*}$ & & & \\
\hline & $\begin{array}{l}\text { Analgesics anti- } \\
\text { pyretic }\end{array}$ & 345 & 23.5 & $\begin{array}{l}58.6 \\
(202)\end{array}$ & 13.7 & $\begin{array}{l}< \\
0.001^{*}\end{array}$ & & & \\
\hline & Oral Steroids & 17 & 1.2 & $58.8(10)$ & 0.55 & 0.456 & & & \\
\hline & Herbal remedies & 443 & 30.2 & $\begin{array}{l}51.2 \\
(227)\end{array}$ & 0.51 & 0.477 & & & \\
\hline \multirow{3}{*}{$\begin{array}{l}\text { My child/ren } \\
\text { are up to } \\
\text { date with } \\
\text { their } \\
\text { vaccines }\end{array}$} & No & 81 & 5.5 & $45.7(37)$ & 1.49 & 0.476 & & & \\
\hline & Yes & 1029 & 70.0 & $\begin{array}{l}49.5 \\
(509)\end{array}$ & & & & & \\
\hline & I don't have children & 334 & 22.7 & $\begin{array}{l}52.4 \\
(175)\end{array}$ & & & & & \\
\hline \multirow[t]{3}{*}{ Smoking } & No & 1196 & 81.4 & $\begin{array}{l}47.3 \\
(566)\end{array}$ & 19.01 & $\begin{array}{l}< \\
0.001^{*}\end{array}$ & & & \\
\hline & Yes & 195 & 13.3 & $\begin{array}{l}62.1 \\
(121)\end{array}$ & & & & & \\
\hline & $\begin{array}{l}\text { I used to smoke but } \\
\text { quit }\end{array}$ & 53 & 3.6 & $64.2(34)$ & & & & & \\
\hline \multirow[t]{4}{*}{ Smoke type } & Cigarettes & 111 & 7.6 & $58.6(65)$ & 3.66 & 0.056 & & & \\
\hline & Shisha & 83 & 5.7 & $65.1(54)$ & 8.16 & $0.004^{*}$ & & & \\
\hline & Midwakh & 8 & 0.5 & $50(4)$ & 0.01 & 0.992 & & & \\
\hline & Vaping & 22 & 1.5 & $59.1(13)$ & 0.76 & 0.381 & & & \\
\hline \multirow[t]{2}{*}{$\begin{array}{l}\text { Smoking } \\
\text { changed }\end{array}$} & Yes & 201 & 13.7 & $\begin{array}{l}58.7 \\
(118)\end{array}$ & 7.73 & $0.005^{\star}$ & & & \\
\hline & No & 1228 & 83.6 & $\begin{array}{l}48.1 \\
(591)\end{array}$ & & & & & \\
\hline \multirow{2}{*}{$\begin{array}{l}\text { Changes } \\
\text { made to } \\
\text { smoking }\end{array}$} & Stopped/Decreased & 73 & 5 & $54.4(49)$ & 2.80 & 0.422 & & & \\
\hline & Started/Increased & 18 & 1.2 & $14.7(13)$ & & & & & \\
\hline \multirow[t]{4}{*}{$\begin{array}{l}\text { Anxiety } \\
\text { (GAD-7) } \\
\text { levels }\end{array}$} & Minimal & 447 & 30.4 & - & & & $3.9(14)$ & 122.19 & $\begin{array}{l}< \\
0.001^{*}\end{array}$ \\
\hline & Mild & 465 & 32.7 & - & & & $9.6(35)$ & & \\
\hline & Moderate & 296 & 20.2 & - & & & $24.5(58)$ & & \\
\hline & Severe & 261 & 17.8 & - & & & $35.3(71)$ & & \\
\hline \multirow[t]{2}{*}{$\begin{array}{l}\text { Difficulty } \\
\text { getting } \\
\text { things done }\end{array}$} & Not difficult at all & 450 & 30.6 & 16.2(73) & 392.41 & $\begin{array}{l}< \\
0.001^{*}\end{array}$ & $4.5(16)$ & 738.01 & $\begin{array}{l}< \\
0.001^{*}\end{array}$ \\
\hline & Somewhat & 747 & 50.9 & $55.3(413)$ & & & $14.6(87)$ & & \\
\hline
\end{tabular}




\begin{tabular}{|c|c|c|c|c|c|c|c|c|c|}
\hline & \multicolumn{3}{|l|}{ Demographics } & \multicolumn{3}{|c|}{ Anxiety GAD-7 Score $(\geq 8)$} & \multicolumn{3}{|c|}{$\begin{array}{l}\text { Reported Children Emotional } \\
\text { SDQ Score (SDQ } \geq 5)\end{array}$} \\
\hline & Very & 186 & 12.7 & $88.7(165)$ & & & $36.2(55)$ & & \\
\hline & Extremely & 86 & 5.9 & $94.2(81)$ & & & $35.7(20)$ & & \\
\hline \multirow[t]{2}{*}{$\begin{array}{l}\text { Anxiety GAD } \\
\text { class }(\geq 8)\end{array}$} & Mild-Minimal & 737 & 50.2 & - & & & $25.4(144)$ & 86.20 & $<.001^{*}$ \\
\hline & High & 732 & 49.8 & - & & & $5.7(34)$ & & \\
\hline \multirow[t]{2}{*}{$\begin{array}{l}\text { SDQ class } \\
(\geq 5)\end{array}$} & Normal & 982 & 84.7 & $43.1(423)$ & 86.3 & $\begin{array}{l}< \\
0.001^{*}\end{array}$ & & & \\
\hline & Abnormal & 178 & 15.3 & $80.9(144)$ & & & & & \\
\hline
\end{tabular}

Anxiety levels (GAD-7 score and SDQ score)

Almost three quarters $(71 \%)$ of our population reported anxiety, and $38 \%$ had moderate to severe anxiety. When we categorized anxiety by high and low based on the GAD-7 cutoff of 8 , half of our participants (49.8\%) reported higher levels of anxiety. Females $(51.7 \%)$ and participants between the ages of 18 and 24 years $(59.8 \%)$ reported greater anxiety. Higher anxiety levels were reported amongst participants with higher levels of education, but differences were not significant. More than half of participants who indicated they were likely to be vaccinated against COVID-19 were more anxious. More than half of parents who indicated they were likely to vaccinate their children with the COVID-19 vaccine had higher anxiety levels. Higher levels of anxiety were reported by asthmatics and those with headache, sore throat or dizziness. Highly anxious participants were more likely to take vitamin C (53.5\%), anti-inflammatory drugs (62.6\%) and analgesics (58.6\%) Participants who had quit smoking had higher anxiety levels. (Table 1).

The highest percentage of reported emotional problems for children was in participants who were both parents and teachers (26.7\%) compared to parents only $(14.6 \%)$ or teachers only $(4.7 \%)$. Participants who were divorced/separated reported higher SDQ scores in their children (25.4\%), compared to those who were married (15.5\%) and school-aged children or adolescents showed significant differences in emotional problems compared to children who were not (17.5\%) and (19.6\%) respectively). Emotional problems were also more common among children attending lower secondary and higher secondary schools. Parents reporting moderate to severe anxiety levels in the GAD-7, also reported higher SDQ scores in their children. A higher percentage of parents of children with emotional problems also reported they found it "Very or extremely" difficult to get things done (36.1\%) (Table 1).

\section{Knowledge, beliefs, hygienic behavior and anxiety}

Overall, participants showed a good knowledge of COVID-19 and the majority were aware that there was no treatment. Participants (83\%) perceived a likelihood of catching COVID-19 with almost half reporting higher levels of anxiety. More than half who believed they would develop severe illness upon contracting the virus reported higher levels of anxiety (Table 2). Almost all participants had made significant changes in their hygienic behavior since the pandemic and reported increased use of hand sanitizer (87\%), washing hands (99\%), wearing facemasks (47\%), and avoiding crowds (96\%), public transportation (98\%) and handshaking (95\%). Significantly higher levels of anxiety were reported amongst participants who always used hand sanitizers and face masks. When behavioral changes were further categorized into two groups, participants who always practiced hygienic behaviors, reported significantly higher levels of anxiety (Table 3). 
Table 2

Prevalence of GAD-7 score $\geq 8$ by knowledge and beliefs related to COVID-19 $(n=1469)$

\begin{tabular}{|c|c|c|c|c|c|c|}
\hline \multirow[t]{2}{*}{ Characteristics } & \multirow[t]{2}{*}{ Category } & \multirow[t]{2}{*}{$\begin{array}{l}\text { Frequency } \\
(\mathrm{n})\end{array}$} & \multirow[t]{2}{*}{$\%$} & $\begin{array}{l}\text { Anxiety GAD-7 Score ( } \geq \\
\text { 8) }\end{array}$ & \multirow[t]{2}{*}{$\begin{array}{l}\text { Chi } \\
\text { Square }\end{array}$} & \multirow[t]{2}{*}{$\begin{array}{l}p- \\
\text { value }\end{array}$} \\
\hline & & & & $\%(n)$ & & \\
\hline \multirow[t]{3}{*}{$\begin{array}{l}\text { No Treatment Available for COVID- } \\
19\end{array}$} & $\begin{array}{l}\text { Don't } \\
\text { know }\end{array}$ & 187 & 12.7 & $54.5(102)$ & \multirow[t]{3}{*}{2.68} & \multirow[t]{3}{*}{0.262} \\
\hline & False & 180 & 12.3 & $46.1(83)$ & & \\
\hline & True & 1102 & 75.0 & $49.6(547)$ & & \\
\hline \multirow[t]{3}{*}{ Sense of Social Responsibility } & $\begin{array}{l}\text { Don't } \\
\text { know }\end{array}$ & 19 & 1.3 & $36.8(7)$ & \multirow[t]{3}{*}{1.36} & \multirow[t]{3}{*}{0.505} \\
\hline & False & 15 & 1.0 & $46.7(7)$ & & \\
\hline & True & 1435 & 97.7 & $50(718)$ & & \\
\hline \multirow[t]{3}{*}{ Likelihood of catching COVID-19 } & $\begin{array}{l}\text { Don't } \\
\text { know }\end{array}$ & 100 & 6.8 & $40.0(40)$ & \multirow[t]{3}{*}{7.26} & \multirow[t]{3}{*}{$0.026^{*}$} \\
\hline & Not likely & 155 & 10.6 & $43.9(68)$ & & \\
\hline & Likely & 1214 & 82.6 & $51.4(624)$ & & \\
\hline \multirow[t]{3}{*}{ Likelihood of surviving COVID-19 } & $\begin{array}{l}\text { Don't } \\
\text { know }\end{array}$ & 113 & 7.7 & $54.0(61)$ & \multirow[t]{3}{*}{3.14} & \multirow[t]{3}{*}{0.208} \\
\hline & Not likely & 50 & 3.4 & $60.0(30)$ & & \\
\hline & Likely & 1306 & 88.9 & $49.1(64)$ & & \\
\hline \multirow[t]{3}{*}{$\begin{array}{l}\text { Likelihood I will develop severe } \\
\text { illness }\end{array}$} & $\begin{array}{l}\text { Don't } \\
\text { know }\end{array}$ & 199 & 13.5 & $48.2(96)$ & \multirow[t]{3}{*}{13.56} & \multirow[t]{3}{*}{$0.001^{*}$} \\
\hline & Not likely & 433 & 29.5 & $43.0(186)$ & & \\
\hline & Likely & 837 & 57.0 & $49.8(732)$ & & \\
\hline
\end{tabular}


Prevalence of GAD-7 score $\geq 8$ by behavior changes taken $(n=1469)$

\begin{tabular}{|c|c|c|c|c|c|c|}
\hline \multirow[t]{2}{*}{ Characteristics } & \multirow[t]{2}{*}{ Category } & \multirow[t]{2}{*}{$\begin{array}{l}\text { Frequency } \\
(\mathrm{n})\end{array}$} & \multirow[t]{2}{*}{$\%$} & $\begin{array}{l}\text { Anxiety GAD-7 } \\
\text { Score }(\geq 8)\end{array}$ & \multirow[t]{2}{*}{$\begin{array}{l}\text { Chi- } \\
\text { Square }\end{array}$} & \multirow[t]{2}{*}{$\begin{array}{l}p- \\
\text { value }^{*}\end{array}$} \\
\hline & & & & $\%(n)$ & & \\
\hline \multirow[t]{3}{*}{ Cover mouth } & Never & 13 & 0.9 & $46.2(6)$ & \multirow[t]{3}{*}{3.03} & \multirow[t]{3}{*}{0.219} \\
\hline & Occasionally & 43 & 2.9 & $62.8(27)$ & & \\
\hline & Most of the time / Always & 1413 & 96.2 & 49.4 (699) & & \\
\hline \multirow[t]{3}{*}{ Use hand sanitizer } & Never & 26 & 1.8 & $26.9(7)$ & \multirow[t]{3}{*}{10.90} & \multirow[t]{3}{*}{$0.004^{\star}$} \\
\hline & Occasionally & 163 & 11.1 & $41.7(68)$ & & \\
\hline & Most of the time / Always & 1280 & 87.1 & $51.3(657)$ & & \\
\hline \multirow[t]{3}{*}{ Washing hands } & Never & 2 & 0.1 & $50.0(1)$ & \multirow[t]{3}{*}{1.13} & \multirow[t]{3}{*}{0.569} \\
\hline & Occasionally & 14 & 1.0 & $35.7(5)$ & & \\
\hline & Most of the time / Always & 1453 & 98.9 & $50.0(726)$ & & \\
\hline \multirow[t]{3}{*}{ Face mask } & Never & 374 & 25.5 & $48.9(183)$ & \multirow[t]{3}{*}{8.84} & \multirow[t]{3}{*}{$0.012^{*}$} \\
\hline & Occasionally & 408 & 27.8 & $44.4(181)$ & & \\
\hline & Most of the time / Always & 687 & 46.8 & $53.6(368)$ & & \\
\hline \multirow[t]{3}{*}{ Avoid crowds } & Never & 6 & 0.4 & $50.0(3)$ & \multirow[t]{3}{*}{0.25} & \multirow[t]{3}{*}{0.882} \\
\hline & Occasionally & 60 & 4.1 & $46.7(28)$ & & \\
\hline & Most of the time / Always & 1403 & 95.5 & $50(701)$ & & \\
\hline \multirow[t]{3}{*}{ Avoid public transport } & Never & 14 & 1.0 & $50.0(7)$ & \multirow[t]{3}{*}{0.17} & \multirow[t]{3}{*}{0.918} \\
\hline & Occasionally & 22 & 1.5 & $45.5(10)$ & & \\
\hline & Most of the time / Always & 1433 & 97.5 & $49.9(715)$ & & \\
\hline \multirow[t]{3}{*}{ Avoid handshaking } & Never & 12 & 0.8 & $66.7(8)$ & \multirow[t]{3}{*}{1.59} & \multirow[t]{3}{*}{0.451} \\
\hline & Occasionally & 62 & 4.2 & $46.8(29)$ & & \\
\hline & Most of the time / Always & 1395 & 94.9 & $49.8(695)$ & & \\
\hline \multirow[t]{2}{*}{$\begin{array}{l}\text { Behavioral changes total } \\
\text { Category }\end{array}$} & $\begin{array}{l}\text { Occasionally exhibiting } \\
\text { behavior changes }\end{array}$ & 604 & 41.1 & $45.4(274)$ & \multirow[t]{2}{*}{8.18} & \multirow[t]{2}{*}{$<0.004^{*}$} \\
\hline & $\begin{array}{l}\text { Always exhibiting behavior } \\
\text { changes }\end{array}$ & 865 & 58.9 & $53.0(458)$ & & \\
\hline
\end{tabular}

Precautionary measures and anxiety

Although most participants felt less anxious with the government's precautionary measures, participants who disagreed reported higher GAD-7 scores for online learning, cancellation of social events and social isolation. Participants who agreed with precautions showed less anxiety than those who disagreed (Table 4). 
Prevalence of GAD-7 score $\geq 8$ by precautionary measures taken $(n=1469)$

\begin{tabular}{|c|c|c|c|c|c|c|}
\hline \multirow[t]{2}{*}{ Characteristics } & \multirow[t]{2}{*}{ Category } & \multirow[t]{2}{*}{$\begin{array}{l}\text { Frequency } \\
\text { (n) }\end{array}$} & \multirow[t]{2}{*}{$\%$} & $\begin{array}{l}\text { Anxiety GAD-7 } \\
\text { Score }(\geq 8)\end{array}$ & \multirow[t]{2}{*}{$\begin{array}{l}\text { Chi } \\
\text { Square }\end{array}$} & \multirow[t]{2}{*}{$\begin{array}{l}p- \\
\text { value }\end{array}$} \\
\hline & & & & $\%(n)$ & & \\
\hline \multirow{3}{*}{$\begin{array}{l}\text { Online learning at educational } \\
\text { institutions }\end{array}$} & Strongly disagree/Disagree & 220 & 15.0 & $65.0(143)$ & \multirow[t]{3}{*}{36.55} & \multirow{3}{*}{$\begin{array}{l}< \\
0.001^{*}\end{array}$} \\
\hline & Neutral & 238 & 16.2 & $57.6(137)$ & & \\
\hline & Strongly agree/Agree & 1011 & 68.8 & $44.7(452)$ & & \\
\hline \multirow[t]{3}{*}{ Airport screening } & Strongly disagree/Disagree & 67 & 4.6 & $61.2(41)$ & \multirow[t]{3}{*}{14.14} & \multirow[t]{3}{*}{$0.001^{*}$} \\
\hline & Neutral & 136 & 9.3 & $62.5(85)$ & & \\
\hline & Strongly agree/ Agree & 1266 & 86.2 & $47.9(606)$ & & \\
\hline \multirow[t]{3}{*}{ Travel bans } & Strongly disagree/Disagree & 65 & 4.4 & $55.4(36)$ & \multirow[t]{3}{*}{11.11} & \multirow[t]{3}{*}{$0.004^{*}$} \\
\hline & Neutral & 79 & 5.4 & $67.1(53)$ & & \\
\hline & Strongly agree/Agree & 1325 & 90.2 & $48.5(643)$ & & \\
\hline \multirow{3}{*}{$\begin{array}{l}\text { Hand sanitizers in public } \\
\text { spaces }\end{array}$} & Strongly disagree/ Disagree & 33 & 2.2 & $51.5(17)$ & \multirow[t]{3}{*}{2.31} & \multirow[t]{3}{*}{0.315} \\
\hline & Neutral & 98 & 6.7 & $57.1(56)$ & & \\
\hline & Strongly agree/ Agree & 1338 & 91.1 & $49.3(659)$ & & \\
\hline \multirow[t]{3}{*}{ Cancellation of social events } & Strongly disagree/Disagree & 34 & 2.3 & $61.8(21)$ & \multirow[t]{3}{*}{7.59} & \multirow[t]{3}{*}{$0.022^{*}$} \\
\hline & Neutral & 50 & 3.4 & $66.0(33)$ & & \\
\hline & Strongly agree / Agree & 1385 & 94.3 & $49.0(678)$ & & \\
\hline \multirow{3}{*}{$\begin{array}{l}\text { Temporary closure of public } \\
\text { places }\end{array}$} & Strongly disagree/Disagree & 47 & 3.2 & $51.1(24)$ & \multirow[t]{3}{*}{1.76} & \multirow[t]{3}{*}{0.415} \\
\hline & Neutral & 51 & 3.5 & $58.8(30)$ & & \\
\hline & Strongly agree/Agree & 1371 & 93.3 & $49.5(678)$ & & \\
\hline \multirow[t]{3}{*}{ Social isolation } & Strongly disagree /Disagree & 49 & 3.3 & $61.2(30)$ & \multirow[t]{3}{*}{7.26} & \multirow[t]{3}{*}{$0.026^{*}$} \\
\hline & Neutral & 82 & 5.6 & $61.0(50)$ & & \\
\hline & Strongly agree/Agree & 1338 & 91.1 & $48.7(652)$ & & \\
\hline \multirow[t]{2}{*}{$\begin{array}{l}\text { Precautionary measures } \\
\text { category }\end{array}$} & $\begin{array}{l}\text { Disagree with precautionary } \\
\text { measures }\end{array}$ & 605 & 41.2 & $54.9(332)$ & \multirow[t]{2}{*}{10.48} & \multirow[t]{2}{*}{$0.001^{*}$} \\
\hline & $\begin{array}{l}\text { Agree with precautionary } \\
\text { measures }\end{array}$ & 864 & 58.8 & $46.3(400)$ & & \\
\hline
\end{tabular}

\section{Worry, fear and anxiety}

The majority of participants felt public fear was justified. However, we found greater anxiety among those who believed that fear caused unnecessary absences from work and school. Whilst most participants worried about catching COVID-19, the majority were more worried about their parents (75\%) or children (65.5\%) catching COVID-19 or transmitting it to someone else if they caught it (64.5\%). Significantly higher GAD-7 scores were found among all participants who agreed they were worried about catching COVID-19, their parents or children catching it, about what would happen if they caught it, about being in social isolation, loss of income and transmitting it to others. When we categorized worry into two groups, "not too worried" and "very worried", we found significantly higher levels of anxiety among participants who reported being very worried (Table 5). Worry in 
parents was associated with SDQ score, and parents with higher scores reported more emotional problems in their children. Parents who were very worried reported significantly higher SDQ scores for their children (Table 5). 
Table 5

Worry about COVID-19 by GAD-7 score and SDQ score $(n=1469)$

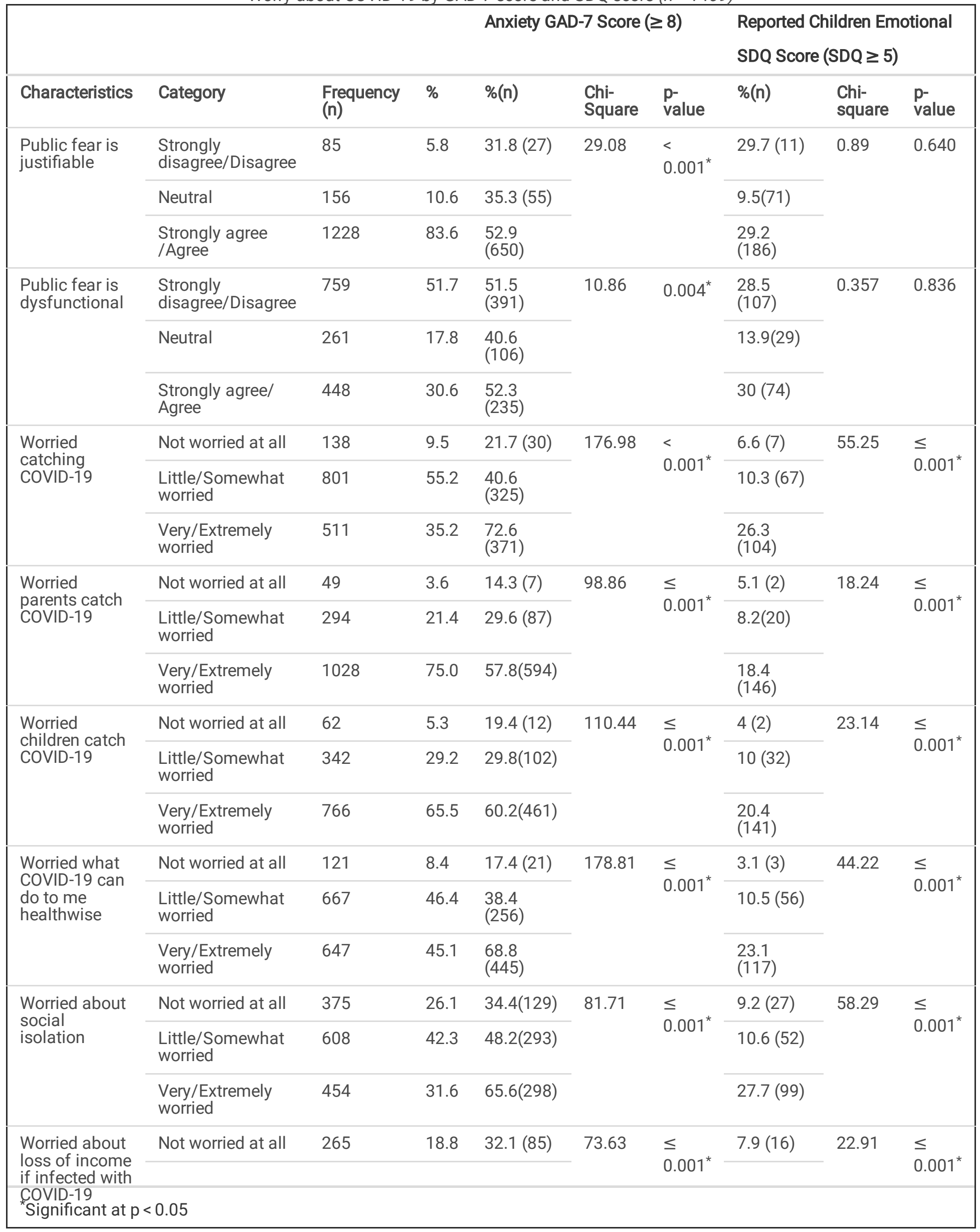




\begin{tabular}{|c|c|c|c|c|c|c|c|c|c|}
\hline & & & & \multicolumn{3}{|c|}{ Anxiety GAD-7 Score $(\geq 8)$} & \multicolumn{3}{|c|}{$\begin{array}{l}\text { Reported Children Emotional } \\
\text { SDQ Score (SDQ } \geq 5)\end{array}$} \\
\hline \multirow{5}{*}{$\begin{array}{l}\text { Worried I } \\
\text { transmit } \\
\text { COVID-19 }\end{array}$} & $\begin{array}{l}\text { Little/Somewhat } \\
\text { worried }\end{array}$ & 441 & 32.9 & $\begin{array}{l}44.4 \\
(196)\end{array}$ & & & $12.1(44)$ & & \\
\hline & $\begin{array}{l}\text { Very/Extremely } \\
\text { worried }\end{array}$ & 633 & 47.3 & $\begin{array}{l}61.6 \\
(390)\end{array}$ & & & $20.7(107)$ & & \\
\hline & Not worried at all & 104 & 8.5 & $31.7(33)$ & 79.81 & $\begin{array}{l}\leq \\
0.001^{*}\end{array}$ & $8.5(8)$ & 32.48 & $\begin{array}{l}\leq \\
0.001^{*}\end{array}$ \\
\hline & $\begin{array}{l}\text { Little/Somewhat } \\
\text { worried }\end{array}$ & 328 & 27.0 & $\begin{array}{l}34.5 \\
(113)\end{array}$ & & & $7.4(20)$ & & \\
\hline & $\begin{array}{l}\text { Very/Extremely } \\
\text { worried }\end{array}$ & 785 & 64.5 & $\begin{array}{l}60.5 \\
(475)\end{array}$ & & & $\begin{array}{l}21.6 \\
(131)\end{array}$ & & \\
\hline \multirow{2}{*}{$\begin{array}{l}\text { Worried in } \\
\text { total about } \\
\text { COVID-19 }\end{array}$} & $\begin{array}{l}\text { Low levels of } \\
\text { worry }\end{array}$ & 394 & 26.8 & $\begin{array}{l}27.4 \\
(108)\end{array}$ & 148.7 & $\begin{array}{l}\leq \\
0.001^{*}\end{array}$ & $6.4(18)$ & 44.9 & $\begin{array}{l}\leq \\
0.001^{*}\end{array}$ \\
\hline & $\begin{array}{l}\text { High levels of } \\
\text { worry }\end{array}$ & 1075 & 73.2 & $58(624)$ & & & $\begin{array}{l}18.2 \\
(160)\end{array}$ & & \\
\hline
\end{tabular}

Among participants with children, most were utilizing effective coping strategies; however, higher anxiety was reported among participants who always openly discussed COVID-19 with their family (51.4\%), compared to those who never did (33.3\%). Participants who always educated their children about proper protective measures (50.3\%) or limited news exposure (53.4\%) had higher anxiety levels $(23.1 \%)$ compared to those who never did these things (41\%). When we categorized these strategies into two groups "occasionally" and "always", we found no differences in anxiety levels based on GAD-7 score. For SDQ scores reported by parents, we found more emotional problems in children whose parents/teachers discussed COVID-19 with them (17.5\%) and among those who educated their children about personal protective measures $(20.9 \%)$. Parents who always utilized coping strategies for dealing with COVID-19 reported children with greater emotional problems than parents who used fewer coping strategies (Table 6). 
Table 6

Coping strategies used with children by GAD-7 score and SDQ score $(n=1469)$

\begin{tabular}{|c|c|c|c|c|c|c|c|c|c|}
\hline \multirow[b]{2}{*}{ Characteristics } & \multirow[b]{2}{*}{ Category } & \multirow[b]{2}{*}{$\begin{array}{l}\text { Frequency } \\
\text { (n) }\end{array}$} & \multirow[b]{2}{*}{$\%$} & \multicolumn{3}{|c|}{ Anxiety GAD-7 Score $(\geq 8)$} & \multicolumn{3}{|c|}{ SDQ Score (SDQ $\geq 5)$} \\
\hline & & & & $\%(n)$ & $\begin{array}{l}\text { Chi- } \\
\text { square }\end{array}$ & $\begin{array}{l}\mathrm{p}- \\
\text { value }\end{array}$ & $\%(n)$ & $\begin{array}{l}\text { Chi- } \\
\text { square }\end{array}$ & $\begin{array}{l}\mathrm{p} \text { - } \\
\text { value }\end{array}$ \\
\hline \multirow{4}{*}{$\begin{array}{l}\text { Openly discuss } \\
\text { COVID-19 with } \\
\text { family }\end{array}$} & No children & 178 & 12.1 & $53.9(96)$ & \multirow[t]{4}{*}{10.79} & \multirow[t]{4}{*}{$0.013^{*}$} & $\begin{array}{l}2.7 \\
(2)\end{array}$ & \multirow[t]{4}{*}{13.42} & \multirow[t]{4}{*}{$0.004^{\star}$} \\
\hline & Never & 27 & 1.8 & $33.3(9)$ & & & $\begin{array}{l}4.2 \\
(1)\end{array}$ & & \\
\hline & Occasionally & 259 & 17.6 & $\begin{array}{l}42.5 \\
(110)\end{array}$ & & & $\begin{array}{l}14.1 \\
(30)\end{array}$ & & \\
\hline & $\begin{array}{l}\text { Most of the } \\
\text { time/Always }\end{array}$ & 1005 & 68.4 & $\begin{array}{l}51.4 \\
(517)\end{array}$ & & & $\begin{array}{l}17.1 \\
(145)\end{array}$ & & \\
\hline \multirow[t]{4}{*}{$\begin{array}{l}\text { Educate children } \\
\text { PPE }\end{array}$} & No children & 316 & 21.5 & $\begin{array}{l}52.2 \\
(165)\end{array}$ & \multirow[t]{4}{*}{10.19} & \multirow[t]{4}{*}{$0.017^{*}$} & $\begin{array}{l}2.4 \\
(3)\end{array}$ & \multirow[t]{4}{*}{21.27} & \multirow[t]{4}{*}{$\begin{array}{l}\leq \\
0.001^{*}\end{array}$} \\
\hline & Never & 13 & 0.9 & $23.1(3)$ & & & $0(0)$ & & \\
\hline & Occasionally & 56 & 3.8 & $33.9(19)$ & & & $\begin{array}{l}10.9 \\
(5)\end{array}$ & & \\
\hline & $\begin{array}{l}\text { Most of the } \\
\text { time/Always }\end{array}$ & 1084 & 73.8 & $\begin{array}{l}50.3 \\
(545)\end{array}$ & & & $\begin{array}{l}17.3 \\
(170)\end{array}$ & & \\
\hline \multirow[t]{4}{*}{$\begin{array}{l}\text { Reassure children } \\
\text { they are safe }\end{array}$} & No children & 331 & 22.5 & $\begin{array}{l}52.6 \\
(174)\end{array}$ & \multirow[t]{4}{*}{1.89} & \multirow[t]{4}{*}{0.595} & $\begin{array}{l}2.3 \\
(3)\end{array}$ & \multirow[t]{4}{*}{23.80} & \multirow[t]{4}{*}{$\begin{array}{l}\leq \\
0.001^{*}\end{array}$} \\
\hline & Never & 20 & 1.4 & $45(9)$ & & & $0(0)$ & & \\
\hline & Occasionally & 92 & 6.3 & $45.7(42)$ & & & $\begin{array}{l}20.3 \\
(16)\end{array}$ & & \\
\hline & $\begin{array}{l}\text { Most of the } \\
\text { time/Always }\end{array}$ & 1026 & 69.8 & $\begin{array}{l}49.4 \\
(507)\end{array}$ & & & $\begin{array}{l}17 \\
(159)\end{array}$ & & \\
\hline \multirow[t]{4}{*}{ Limit news exposure } & No children & 407 & 27.7 & $\begin{array}{l}50.9 \\
(207)\end{array}$ & \multirow[t]{4}{*}{11.20} & \multirow[t]{4}{*}{$0.011^{*}$} & $3(6)$ & \multirow[t]{4}{*}{37.10} & \multirow[t]{4}{*}{$\begin{array}{l}\leq \\
0.001^{*}\end{array}$} \\
\hline & Never & 205 & 14.0 & $41(84)$ & & & $\begin{array}{l}11.2 \\
(21)\end{array}$ & & \\
\hline & Occasionally & 216 & 14.7 & $45.8(99)$ & & & $\begin{array}{l}22.1 \\
(43)\end{array}$ & & \\
\hline & $\begin{array}{l}\text { Most of the } \\
\text { time/Always }\end{array}$ & 641 & 43.6 & $\begin{array}{l}53.4 \\
(342)\end{array}$ & & & $\begin{array}{l}18.6 \\
(108)\end{array}$ & & \\
\hline \multirow[t]{4}{*}{$\begin{array}{l}\text { Create schedule } \\
\text { learning }\end{array}$} & No children & 335 & 22.8 & $\begin{array}{l}48.4 \\
(162)\end{array}$ & \multirow[t]{4}{*}{0.51} & \multirow[t]{4}{*}{0.916} & $\begin{array}{l}6.6 \\
(10)\end{array}$ & \multirow[t]{4}{*}{10.40} & \multirow[t]{4}{*}{$0.015^{\star}$} \\
\hline & Never & 104 & 7.1 & $51(53)$ & & & $\begin{array}{l}17.4 \\
(16)\end{array}$ & & \\
\hline & Occasionally & 288 & 19.6 & $51(147)$ & & & $\begin{array}{l}16.4 \\
(42)\end{array}$ & & \\
\hline & $\begin{array}{l}\text { Most of the } \\
\text { time/Always }\end{array}$ & 742 & 50.5 & $\begin{array}{l}49.9 \\
(370)\end{array}$ & & & $\begin{array}{l}16.7 \\
(111)\end{array}$ & & \\
\hline $\begin{array}{l}\text { Maintain regular } \\
\text { routine }\end{array}$ & No children & 225 & 15.3 & $\begin{array}{l}50.7 \\
(114)\end{array}$ & 2.26 & 0.521 & $\begin{array}{l}4.4 \\
(4)\end{array}$ & 8.99 & $0.029^{*}$ \\
\hline gnificant at $p<0$ & & & & & & & & & \\
\hline
\end{tabular}




\begin{tabular}{|c|c|c|c|c|c|c|c|c|c|}
\hline & & & & \multicolumn{3}{|c|}{ Anxiety GAD-7 Score $(\geq 8)$} & \multicolumn{3}{|c|}{ SDQ Score (SDQ $\geq 5)$} \\
\hline & Never & 47 & 3.2 & $46.8(22)$ & & & $\begin{array}{l}14.7 \\
(5)\end{array}$ & & \\
\hline & Occasionally & 185 & 12.6 & $\begin{array}{l}54.6 \\
(101)\end{array}$ & & & $\begin{array}{l}16.2 \\
(25)\end{array}$ & & \\
\hline & $\begin{array}{l}\text { Most of the } \\
\text { time/Always }\end{array}$ & 1012 & 68.9 & $\begin{array}{l}48.9 \\
(495)\end{array}$ & & & $\begin{array}{l}16.3 \\
(144)\end{array}$ & & \\
\hline \multirow[t]{2}{*}{$\begin{array}{l}\text { Coping strategies } \\
\text { total Category }\end{array}$} & $\begin{array}{l}\text { Low use of } \\
\text { coping } \\
\text { strategies }\end{array}$ & 705 & 48.0 & $49.7(366)$ & 1.65 & \multirow[t]{2}{*}{0.199} & $\begin{array}{l}5.6 \\
(10)\end{array}$ & 9.01 & \multirow[t]{2}{*}{$\begin{array}{l}< \\
0.001^{*}\end{array}$} \\
\hline & $\begin{array}{l}\text { High use of } \\
\text { coping } \\
\text { strategies }\end{array}$ & 765 & 52.0 & $50.3(371)$ & & & $\begin{array}{l}17.1 \\
(168)\end{array}$ & & \\
\hline
\end{tabular}

To estimate the probability of anxiety levels among participants in our study, two multivariate logistic regressions were conducted-one with the GAD-7 score $\geq 8$ as a measure of adult anxiety and the other with the SDQ score $\geq 5$ for anxiety and emotional problems in children. In the first model, the effects of gender, age, age of children, perception of fear, perception of likelihood to contract COVID-19 and to develop severe disease, headaches, sore throat, asthma, measures taken for symptoms, smoking, and changed smoking habits, likelihood of vaccination for self and children, hygienic behavior category, precautionary measures category and worry category were modelled. The omnibus model for logistic regression analysis was statistically significant. The model explained $28 \%$ of the variance in anxiety levels. Hosmer and Lemeshow test results confirmed the model was a good fit for the data (Table 7). Females had 1.91 times higher odds of reporting anxiety than males, and participants who believed that fear was justified were six times more anxious than those who disagreed. Higher levels of worry were also associated with increased anxiety levels. Participants who said they would take the COVID-19 vaccine were 1.57 times more likely to report higher anxiety, however, vaccinating children did not influence anxiety. The odds of higher anxiety were larger among participants who smoked, took vitamin C for symptoms and reported sore throat (Table 7). 
Table 7

Multivariate analysis for variables predicting anxiety (GAD-7 score $\geq 8)$ in sample population and variables predicting parent/teacher reported emotional problems in children (SDQ score) $(n=1469)$

\begin{tabular}{|c|c|c|c|c|c|}
\hline \multicolumn{6}{|c|}{ Generalized Anxiety Disorder (GAD-7) score } \\
\hline Variable & & $\mathrm{b}$ & $\operatorname{SE}(b)$ & P-value & $\begin{array}{l}\mathrm{OR}[95 \% \\
\mathrm{Cl}]\end{array}$ \\
\hline \multirow[t]{2}{*}{ Gender } & Female & 0.649 & 0.178 & $<0.001$ & $\begin{array}{l}1.91 \\
{[1.35-} \\
2.71]\end{array}$ \\
\hline & Male $^{a}$ & - & - & - & 1 \\
\hline \multirow[t]{2}{*}{ Precautionary Measures } & Agree & -0.740 & 0.146 & $<0.001$ & $\begin{array}{l}0.48 \\
{[0.36-} \\
0.63]\end{array}$ \\
\hline & Disagree $^{a}$ & - & - & - & 1 \\
\hline \multirow[t]{2}{*}{ Public fear Justifiable } & Agree & 1.811 & 1.082 & 0.094 & $\begin{array}{l}6.11[0.73- \\
51.0]\end{array}$ \\
\hline & Disagree $^{a}$ & - & - & - & 1 \\
\hline \multirow[t]{2}{*}{ Worry associated with COVID-19 } & High levels of worry & 1.336 & 0.139 & $<0.001$ & $\begin{array}{l}3.80[2.90- \\
5.00]\end{array}$ \\
\hline & Low levels of worry ${ }^{a}$ & - & - & - & 1 \\
\hline \multirow[t]{2}{*}{$\begin{array}{l}\text { Will take } \\
\text { CoVID-19 Vaccine }\end{array}$} & Yes & 0.446 & 0.1478 & 0.003 & $\begin{array}{l}1.57 \\
{[1.17-} \\
2.09]\end{array}$ \\
\hline & $\mathrm{No}^{\mathrm{a}}$ & - & - & - & 1 \\
\hline \multirow[t]{2}{*}{$\begin{array}{l}\text { Symptoms- } \\
\text { Sore throat }\end{array}$} & Yes & 0.447 & 0.173 & 0.010 & $\begin{array}{l}1.56 \\
{[1.17-} \\
2.09]\end{array}$ \\
\hline & $\mathrm{No}^{\mathrm{a}}$ & - & - & - & 1 \\
\hline \multirow[t]{2}{*}{ Taking Vitamin $\mathrm{C}$ as measure } & Yes & 0.344 & 0.134 & 0.010 & $\begin{array}{l}1.41 \\
{[1.09-} \\
1.83]\end{array}$ \\
\hline & $\mathrm{No}^{\mathrm{a}}$ & - & - & - & 1 \\
\hline \multirow[t]{2}{*}{ Smoker } & Yes & 0.435 & 0.194 & 0.025 & $\begin{array}{l}1.55 \\
{[1.06-} \\
2.26]\end{array}$ \\
\hline & $\mathrm{No}^{\mathrm{a}}$ & - & - & - & 1 \\
\hline
\end{tabular}

Likelihood Ratio Chi-Square $276.2(\mathrm{df}=40$, $\mathrm{p}$-value $\leq$ 0.001)

Strengths and Difficulties Questionnaire (SDQ) score

\begin{tabular}{llllll} 
Variable & & $\mathrm{b}$ & $\mathrm{SE}(\mathrm{b})$ & P-value & OR [95\% Cl] \\
\hline Relationship to child/ren & Parent only & 0.854 & 0.493 & 0.884 & $\begin{array}{l}2.35[0.89- \\
6.17]\end{array}$ \\
\cline { 2 - 5 } & $\begin{array}{l}\text { Parent \& } \\
\text { Teacher }\end{array}$ & 1.626 & 0.519 & $<0.001$ & $5.08[1.84-14.0]$
\end{tabular}

${ }^{a}$ reference group, $b$ parameter estimate, SE Std Error, OR Odds Ratio, C/ Confidence Interval 


\begin{tabular}{|c|c|c|c|c|c|}
\hline \multicolumn{6}{|l|}{ Generalized Anxiety Disorder (GAD-7) score } \\
\hline & Teacher only ${ }^{a}$ & - & - & - & 1 \\
\hline \multirow[t]{2}{*}{ Child/ren in lower secondary education } & Yes & 0.522 & 0.189 & 0.006 & $\begin{array}{l}1.69[1.16- \\
2.44]^{-1}\end{array}$ \\
\hline & No ${ }^{a}$ & - & - & - & 1 \\
\hline \multirow[t]{2}{*}{$\begin{array}{l}\text { Child/ren in higher secondary } \\
\text { education }\end{array}$} & Yes & 0.460 & 0.199 & 0.021 & $\begin{array}{l}1.59[1.07- \\
2.34]\end{array}$ \\
\hline & $\mathrm{No}^{\mathrm{a}}$ & - & - & - & 1 \\
\hline \multirow[t]{4}{*}{ Anxiety level (GAD-7) } & Severe & 1.94 & 0.355 & $<0.001$ & $7.00[3.45-14.0]$ \\
\hline & Moderate & 1.505 & 0.340 & 0.013 & $\begin{array}{l}4.51[2.31- \\
8.80]\end{array}$ \\
\hline & Mild & 0.582 & 0.344 & 0.011 & $\begin{array}{l}1.79[0.91- \\
3.50]\end{array}$ \\
\hline & Minimal $^{\mathrm{a}}$ & - & - & - & 1 \\
\hline \multirow[t]{4}{*}{$\begin{array}{l}\text { Difficulty of parent/teacher to get } \\
\text { things done }\end{array}$} & Extremely & 1.299 & 0.439 & 0.003 & $\begin{array}{l}3.70[1.55- \\
8.66]\end{array}$ \\
\hline & Very & 1.403 & 0.348 & $<0.001$ & $\begin{array}{l}4.07[2.10- \\
8.05]\end{array}$ \\
\hline & Somewhat & 0.805 & 0.306 & 0.009 & $\begin{array}{l}2.24[1.23- \\
4.08]\end{array}$ \\
\hline & $\begin{array}{l}\text { Not difficult at } \\
\text { all }^{\text {a }}\end{array}$ & - & - & - & 1 \\
\hline \multicolumn{6}{|c|}{ Likelihood Ratio Chi-Square 185.90 (df = 17, p-value $\leq 0.001)$} \\
\hline
\end{tabular}

In the second model, with SDG $\geq 5$ as a measure of anxiety in children, the effects of relationship, age (school-aged or adolescent), marital status, educational level of child (lower secondary and higher secondary), coping strategies, worry, parental anxiety level and difficulty getting things done were modelled. The omnibus model for logistic regression analysis was significant and explained $26 \%$ of the variance in children's anxiety levels. Hosmer and Lemeshow test results confirmed the model was a good fit, (Table 7). Participants who were both parents and teachers were five times more likely to report emotional problems in children mostly in adolescents in lower and secondary school. Parents with severe anxiety levels were seven times more likely to report emotional problems in their children. Parental reports of "finding it very difficult to do work, to do things at home and to get along with other people" were a strong predictor of emotional problems in children (Table 7).

\section{Discussion}

This study revealed that the pandemic has had a significant impact on the mental health and well-being of the UAE population with the majority of study participants reporting mild to severe anxiety. This was most prevalent among women suggesting that they suffered psychologically more from the pandemic than males [33, 34]. Their anxiety may be exacerbated by the added responsibility of home schooling, work commitments, social isolation and increased concern for family and loved ones. Our study found that government measures to contain the virus largely decreased anxiety. Many reported anxiety about online learning disrupting their children's education and examinations. Airport closures and travel bans are major anxiety producers because the UAE is a popular travel hub. The potential loss of tourism jobs, inability to travel to family and loved ones and overall loss of connection with the world causes worry[35-37]. Greater worry was correlated with higher GAD-7 scores for 
concerns over parents' and children's health, fears of bringing infection home from the workplace and loss of income. Parental worry seemed associated with emotional problems in children, but further analysis showed lower correlation.

Perceptions of greater risk corresponded to increased anxiety. These findings contradicted research conducted in China during the early stages of the pandemic where participants reported lower perceived likelihood of contracting COVID-19, which was associated with lower stress [11]. High-risk perception tends to increase protective and hygienic behaviors such as handwashing and social-distancing $[38,39]$. In our study, the majority who had higher GAD-7 scores reported wearing masks and using hand sanitizers. As demonstrated in our study, those with health conditions like asthma were more likely to feel concern because of probability of infection [40]. Sore throat, taking vitamin C and smoking remained significant predictors of anxiety levels among participants in our study upon further analysis. Smokers are at greater risk of developing severe COVID-19 [41-43].

The majority of our study population intended to take the COVID-19 vaccine when available and vaccinate their children, although a relatively large percentage said they would not take the vaccine. This is similar to a recent study in France [44] and a local study showing $12 \%$ vaccine hesitancy among the UAE population [45]. Hesitancy about the vaccine was mainly related to safety and political concerns $[44,46]$. Governments need to publicize the measures taken to ensure vaccine safety.

The psychological impact of COVID-19 on children was assessed for the first time in our study. COVID-19 adversely affects the mental health of children, particularly those in lower grades. Social isolation, prolonged school closure, challenges with online learning and uncertainty over assessments and examinations all cause mental stress, especially among adolescents [47]. Although we did not interview children directly, we used parent/teacher questionnaires, which were validated against structured diagnostic interviews. Parents and teachers, and especially parents who are teachers, were the best informants of emotional problems in children. Parents who regularly utilized coping strategies with their children reported higher SDQ scores than those who did not. Further research should measure the effectiveness of these strategies in addressing anxiety disorders in children. We found that higher parental anxiety was a significant predictor of children's SDQ score, suggesting a significant association between parental and child anxiety. This is consistent with research where mental health service utilization among adolescents was associated with parental anxiety and depression [13]. Our study suggests that parental anxiety might be a unique factor in explaining anxiety disorders in children and should be included in future research on the psychological impact of public health emergencies in this population.

\section{Limitations}

The use of convenience sampling and its descriptive nature through an online survey may not allow the generalization of results. However, considering the need for a quick method to assess the psychological impact on a population during a rapidly evolving infectious disease outbreak, the online survey proved best [48]. Responses were collected from all over the UAE in addition to countries outside the UAE (due to online and social media use) with a good response rate allowing for some degree of representativeness. The self-reported data in the survey may lead to response biases specifically for reported behavioral changes, coping strategies and measures taken where participants provide socially desirable results. Self-reported levels of anxiety among adults and emotional disorders in children may not be as accurate as those assessed by healthcare professionals.

\section{Conclusion}

This is the first study to provide information on the psychological impact of COVID-19 on parents and children, with association between parental and child anxiety. Worry and fear are significant predictors of growing anxiety in the UAE. Policymakers should use the findings to develop effective screening methods and interventions to improve mental health, especially for children. Such strategies can reduce the psychological impact of COVID-19 and other public health emergencies in the future.

\section{Declarations}

Ethics approval and consent to participate 
All procedures performed in the study were in accordance with the ethical standards of the institutional and/or national research committee and with the 1964 Helsinki declaration and its later amendments or comparable ethical standards. This study protocol was evaluated and approved by the University of Sharjah and the United Arab Emirates University ethical review boards. Written consent was obtained prior to individual participation.

$\underline{\text { Consent for publication }}$

Not applicable

Availability of data and materials

The datasets used and/or analysed during the current study are available from the corresponding author on reasonable request.

Competing interests

The authors declare that they have no competing interests

Funding

This research received no specific grant from any funding agency, commercial or not-for-profit sectors.

Authors' contributions

$B S, R H, F S$, and MT conceived, designed and initiated the study. AA, IE, AH, ES contributed to the planning and implementation of the study. AAS analyzed survey data. BS, AAS interpreted the results. BS drafted the manuscript with input from RH, IE, AA, FS, $\mathrm{AH}, \mathrm{MT}, \mathrm{ES}$ and QH. All authors read and approved the final version of the manuscript.

Acknowledgements The authors would like to thank all the individuals who generously shared their time and took part in the study.

Supplementary Material

The survey used to collect data for this study is provided as supplementary material.

\section{References}

1. WHO. Coronavirus disease (COVID-19) outbreak 2020 [Available from: https://covid19.who.int/.

2. WHO. Director General's opening remarks at the media briefing on COVID-19, 11th March 20202020 [updated 30-3-2020. Available from: https://www.who.int/dg/speeches/detail/who-director-general-s-opening-remarks-at-the-media-briefing-oncovid-19-11-march-2020.

3. Worldometers.info. COVID-19 CORONAVIRUS PANDEMIC. Dover, Delaware, U.S.A. 2020 [Available from: https://www.worldometers.info/coronavirus/.

4. Wang C, Horby PW, Hayden FG, Gao GF. A novel coronavirus outbreak of global health concern. Lancet (London, England). 2020;395(10223):470-3.

5. Rubin GJ, Wessely S. The psychological effects of quarantining a city. British Medical Journal. 2020;368:m313.

6. Sim K, Huak Chan Y, Chong PN, Chua HC, Wen Soon S. Psychosocial and coping responses within the community health care setting towards a national outbreak of an infectious disease. Journal of psychosomatic research. 2010;68(2):195-202.

7. Balkhy HH, Abolfotouh MA, Al-Hathlool RH, Al-Jumah MA. Awareness, attitudes, and practices related to the swine influenza pandemic among the Saudi public. BMC infectious diseases. 2010;10:42.

8. Lau JT, Yang X, Tsui HY, Pang E. SARS related preventive and risk behaviours practised by Hong Kong-mainland China cross border travellers during the outbreak of the SARS epidemic in Hong Kong. Journal of epidemiology and community health. 2004;58(12):988-96.

Page 22/ 24 
9. Leung GM, Lam TH, Ho LM, Ho SY, Chan BH, Wong IO, et al. The impact of community psychological responses on outbreak control for severe acute respiratory syndrome in Hong Kong. Journal of epidemiology and community health.

2003;57(11):857-63.

10. Huang Y, Zhao N. Generalized anxiety disorder, depressive symptoms and sleep quality during COVID-19 outbreak in China: a web-based cross-sectional survey. Psychiatry research. 2020;288:112954.

11. Wang C, Pan R, Wan X, Tan Y, Xu L, Ho CS, et al. Immediate Psychological Responses and Associated Factors during the Initial Stage of the 2019 Coronavirus Disease (COVID-19) Epidemic among the General Population in China. International journal of environmental research and public health. 2020;17(5).

12. Shekerdemian LS, Mahmood NR, Wolfe KK, Riggs BJ, Ross CE, McKiernan CA, et al. Characteristics and Outcomes of Children With Coronavirus Disease 2019 (COVID-19) Infection Admitted to US and Canadian Pediatric Intensive Care Units. JAMA pediatrics. 2020.

13. Essau CA. Frequency and patterns of mental health services utilization among adolescents with anxiety and depressive disorders. Depression and anxiety. 2005;22(3):130-7.

14. Jongerden L, Simon E, Bodden DH, Dirksen CD, Bogels SM. Factors associated with the referral of anxious children to mental health care: the influence of family functioning, parenting, parental anxiety and child impairment. International Journal of Methods in Psychiatric Research. 2015;24(1):46-57.

15. Boman F, Stafström M, Lundin N, Moghadassi M, Törnhage CJ, Östergren PO. Comparing parent and teacher assessments of mental health in elementary school children. Scandinavian journal of public health. 2016;44(2):168-76.

16. Goodman R, Ford T, Simmons H, Gatward R, Meltzer H. Using the Strengths and Difficulties Questionnaire (SDQ) to screen for child psychiatric disorders in a community sample. International Review of Psychiatry. 2003;15(1-2):166-72.

17. Aelterman A, Engels N, Van Petegem K, Pierre Verhaeghe J. The well-being of teachers in Flanders: the importance of a supportive school culture. Educational Studies. 2007;33(3):285-97.

18. Telles S, Gupta RK, Bhardwaj AK, Singh N, Mishra P, Pal DK, et al. Increased Mental Well-Being and Reduced State Anxiety in Teachers After Participation in a Residential Yoga Program. Medical science monitor basic research. 2018;24:105-12.

19. SurveyMonkey. San Mateo [Available from: www.surveymonkey.com.

20. Al-Rabiaah A, Temsah MH, Al-Eyadhy AA, Hasan GM, Al-Zamil F, Al-Subaie S, et al. Middle East Respiratory SyndromeCorona Virus (MERS-CoV) associated stress among medical students at a university teaching hospital in Saudi Arabia. Journal of infection and public health. 2020.

21. Bults M, Beaujean DJ, Richardus JH, Voeten HA. Perceptions and behavioral responses of the general public during the 2009 influenza A (H1N1) pandemic: a systematic review. Disaster medicine and public health preparedness. 2015;9(2):207-19.

22. DeCoster J, Gallucci M, Iselin A-MR. Best Practices for Using Median Splits, Artificial Categorization, and their Continuous Alternatives. Journal of Experimental Psychopathology. 2011;2(2):197-209.

23. Leung GM, Ho LM, Chan SK, Ho SY, Bacon-Shone J, Choy RY, et al. Longitudinal assessment of community psychobehavioral responses during and after the 2003 outbreak of severe acute respiratory syndrome in Hong Kong. Clinical Infectious Diseases. 2005;40(12):1713-20.

24. Spitzer RL, Kroenke K, Williams JB, Lowe B. A brief measure for assessing generalized anxiety disorder: the GAD-7. Archives of internal medicine. 2006;166(10):1092-7.

25. Kroenke K, Spitzer RL, Williams JB, Monahan PO, Lowe B. Anxiety disorders in primary care: prevalence, impairment, comorbidity, and detection. Annals of internal medicine. 2007;146(5):317-25.

26. Goodman R. The Strengths and Difficulties Questionnaire: a research note. Journal of Child Psychology and Psychiatry. 1997;38(5):581-6.

27. Goodman A, Goodman R. Strengths and difficulties questionnaire as a dimensional measure of child mental health. Journal of the American Academy of Child and Adolescent Psychiatry. 2009;48(4):400-3.

28. Silva TBF, Osório FL, Loureiro SR. SDQ: discriminative validity and diagnostic potential. Frontiers in Pyschology. 2015;6(811). 
29. Goodman A. Scoring the Strengths \& Difficulties Questionnaire for age 4-17 [Available from: https://www.ehcap.co.uk/content/sites/ehcap/uploads/NewsDocuments/236/SDQEnglishUK4-17scoring-1.PDF.

30. Alyahri A, Goodman R. Validation of the Arabic Strengths and Difficulties Questionnaire and the Development and WellBeing Assessment. Eastern Mediterranean Health Journal. 2006;12 Suppl 2:S138-46.

31. Sawaya $H$, Atoui M, Hamadeh A, Zeinoun P, Nahas Z. Adaptation and initial validation of the Patient Health Questionnaire - 9 (PHQ-9) and the Generalized Anxiety Disorder - 7 Questionnaire (GAD-7) in an Arabic speaking Lebanese psychiatric outpatient sample. Psychiatry research. 2016;239:245-52.

32. SAS Institute Inc. 2011. Base SAS® 9.3 Procedures Guide. Cary NSII.

33. Lim GY, Tam WW, Lu Y, Ho CS, Zhang MW, Ho RC. Prevalence of Depression in the Community from 30 Countries between 1994 and 2014. Scientific reports. 2018;8(1):2861.

34. Löwe B, Decker O, Müller S, Brähler E, Schellberg D, Herzog W, et al. Validation and standardization of the Generalized Anxiety Disorder Screener (GAD-7) in the general population. Medical care. 2008;46(3):266-74.

35. Borkovec TD, Ray WJ, Stöber J. Worry: A Cognitive Phenomenon Intimately Linked to Affective, Physiological and Interpersonal Behavioral Processes. Cognitive Therapy and Research. 1998;22(6):561-76.

36. Paulesu E, Sambugaro E, Torti T, Danelli L, Ferri F, Scialfa G, et al. Neural correlates of worry in generalized anxiety disorder and in normal controls: a functional MRI study. Psychological medicine. 2010;40(1):117-24.

37. Sun X, So SH, Chan RCK, Chiu CD, Leung PWL. Worry and metacognitions as predictors of the development of anxiety and paranoia. Scientific reports. 2019;9(1):14723.

38. Harper CA, Satchell LP, Fido D, Latzman RD. Functional Fear Predicts Public Health Compliance in the COVID-19 Pandemic. International journal of mental health and addiction. 2020:1-14.

39. Motta Zanin G, Gentile E, Parisi A, Spasiano D. A Preliminary Evaluation of the Public Risk Perception Related to the COVID19 Health Emergency in Italy. International Journal of Environmental Research and Public Health. 2020;17(9).

40. Liao Q, Cowling BJ, Lam WW, Ng DM, Fielding R. Anxiety, worry and cognitive risk estimate in relation to protective behaviors during the 2009 influenza A/H1N1 pandemic in Hong Kong: ten cross-sectional surveys. BMC infectious diseases. 2014;14:169.

41. Eisenberg SL, Eisenberg MJ. Smoking Cessation During the COVID-19 Epidemic. Nicotine and Tobacco Research. 2020.

42. Heerfordt C, Heerfordt IM. Has there been an increased interest in smoking cessation during the first months of the COVID-19 pandemic? A Google Trends study. Public health. 2020;183:6-7.

43. Vardavas $\mathrm{Cl}$, Nikitara K. COVID-19 and smoking: A systematic review of the evidence. Tobacco induced diseases. 2020;18:20.

44. de Girolamo G, Cerveri G, Clerici M, Monzani E, Spinogatti F, Starace F, et al. Mental Health in the Coronavirus Disease 2019 Emergency-The Italian Response. JAMA psychiatry. 2020.

45. Alsuwaidi AR, Elbarazi I, Al-Hamad S, Aldhaheri R, Sheek-Hussein M, Narchi H. Vaccine hesitancy and its determinants among Arab parents: a cross-sectional survey in the United Arab Emirates. Human Vaccines and Immunotherapeutics. 2020:1-7.

46. Harrison EA, Wu JW. Vaccine confidence in the time of COVID-19. European journal of epidemiology. 2020;35(4):325-30.

47. Al-Yateem N, Bani Issa W, Rossiter RC, Al-Shujairi A, Radwan H, Awad M, et al. Anxiety related disorders in adolescents in the United Arab Emirates: a population based cross-sectional study. BMC pediatrics. 2020;20(1):245.

48. Geldsetzer P. Use of Rapid Online Surveys to Assess People's Perceptions During Infectious Disease Outbreaks: A Crosssectional Survey on COVID-19. Journal of medical Internet research. 2020;22(4):e18790.

\section{Supplementary Files}

This is a list of supplementary files associated with this preprint. Click to download.

- ParentsAnxietySurveySubmission.pdf

Page 24/24 